Exercices Exercices de rhétorique

de rhétorique $\quad 9 \mid 2017$

Sur la consolation

\title{
Présentation : consolation et rhétorique
}

\section{Claudie Martin-Ulrich}

\section{(2) OpenEdition}

Journals

Édition électronique

URL : http://journals.openedition.org/rhetorique/543

DOI : 10.4000/rhetorique.543

ISSN : 2270-6909

\section{Éditeur}

UGA Éditions/Université Grenoble Alpes

\section{Édition imprimée}

ISBN : 978-2-37747-010-5

\section{Référence électronique}

Claudie Martin-Ulrich, "Présentation : consolation et rhétorique », Exercices de rhétorique [En ligne], 9| 2017, mis en ligne le 22 juin 2017, consulté le 12 septembre 2020. URL : http://

journals.openedition.org/rhetorique/543 ; DOI : https://doi.org/10.4000/rhetorique.543

Ce document a été généré automatiquement le 12 septembre 2020

\section{(c) (i) (2)}

Les contenus de la revue Exercices de rhétorique sont mis à disposition selon les termes de la Licence Creative Commons Attribution - Pas d'Utilisation Commerciale - Partage dans les Mêmes Conditions 4.0 International. 


\title{
Présentation : consolation et rhétorique
}

\author{
Claudie Martin-Ulrich
}

1 Pour reprendre un titre moderne, "notre besoin de consolation est insatiable ${ }^{1}$ ", or ce besoin est sans doute aujourd'hui pris en charge par les sciences sociales notamment par le care, ou par la psychologie et la psychanalyse avec le travail de deuil ${ }^{2}$. Mais la forme même que prend la consolation est sortie du champ de la réflexion alors même qu'elle était autrefois un élément fondamental. En 1992 déjà, Ilsetraut Hadot ouvrait la préface de son édition des Consolations de Sénèque en constatant la disparition du genre de la consolation ${ }^{3}$. Une nouvelle étape semble franchie depuis, puisqu'associer l'acte de consoler à un type spécifique de discours n'est plus ou pas un réflexe ${ }^{4}$. On pense couramment que, produite par un élan du cœur, la consolation est spontanée donc désordonnée, ou du moins sans forme préétablie possible, sans plan ni patron. En d'autres termes, si la pratique de la consolation n'a pas disparu, c'est le lien entre consolation et rhétorique qui s'est évanoui.

2 Ces quelques remarques suffisent à situer l'ambition de ce numéro. Il s'agit d'étudier la consolation selon un spectre temporel large - de l'Antiquité à la fin du XVII ${ }^{\mathrm{e}}$ siècle ${ }^{5}-$, c'est-à-dire à une époque où le lien avec la rhétorique relevait de l'évidence partagée. Le problème est alors celui de l'immensité du corpus, qui n'est pas seulement textuel. On consolait par la parole, mais aussi par le geste. Le vaste corpus consolatoire contient donc des discours (eux-mêmes de nature très variée) et des pratiques, elles-mêmes connues grâce à des sources textuelles et, dans une moindre mesure, par l'iconographie. Ce vaste corpus est de plus résistant, puisqu'en dehors des grands textes classiques bien identifiés par la tradition littéraire et philosophique, il existe toute une série de discours consolatoires qui eux-mêmes se développent sous des formes très diverses pour ne pas dire tentaculaires. D'un côté, émergent des textes autonomes, souvent intitulés "consolations» et formant un genre véritable: le genre de la consolation, bien représenté par les lettres, mais pas uniquement. Bénéficiant d'une certaine notoriété ou d'une visibilité bien marquée, ce premier massif volumineux et foisonnant s'identifie sans trop de difficulté. D'un autre côté, on peut repérer de larges 
parcelles relevant du corpus consolatoire par leur thème, mais qui apparaissent de façon beaucoup plus éclatée, dans la littérature comme dans des sources non fictionnelles ${ }^{6}$.

3 De surcroît, les formes du discours sur la consolation que l'on vient de cerner rapidement mobilisent un troisième sous-groupe de textes: le vaste territoire des genres non fictionnels où se lisent les pratiques normées de l'acte de consoler, pratiques qui peuvent varier selon les époques et les lieux. Les correspondances privées ou publiques, la littérature de dévotion comme les manuels à l'usage des prêtres ou des pasteurs, les livres de piété, les récits de mort, les recueils de poésie funèbre, les sermons, pour ne citer que quelques exemples significatifs, présentent des descriptions essentielles pour saisir la pratique consolatoire d'une époque.

4 L'immensité du corpus se retrouve du côté de la théorie. Les manuels de rhétorique et d'épistolographie, en décrivant chaque type de discours, contiennent des sources abondantes de réflexions sur l'acte de consoler, d'autant plus que ces théoriciens exposent et commentent les pratiques contemporaines en fonction des connaissances de la psyché sur lesquelles se moulent leurs descriptions. Les traités rendent en particulier explicites les liens hiérarchiques comme les liens d'amitié qui unissaient entre eux les individus ${ }^{7}$ : au-delà de la psyché d'une époque, on voit ainsi apparaître les valeurs sur lesquelles se fondait telle ou telle société ancienne.

5 La définition du corpus consolatoire, copieux et protéiforme, est donc un défi, et son étude ne saurait se limiter en soi à une analyse relevant de la seule rhétorique. Celle-ci nous servira pourtant de fil rouge, car c'est peut-être là que s'expriment le mieux les problèmes que soulève l'idée même de consoler. De l'Antiquité à la fin du XVII siècle, nombre de discours se sont insurgé contre la possibilité de mettre fin à la souffrance par la seule force de la parole d'un consolateur. La question, autant rhétorique qu'éthique, est centrale. La consolation met l'orateur au défi : peut-on vraiment apaiser la douleur, éradiquer la mélancolie, soulager la peine par des mots? L'intention de consoler suffit-elle face à l'anéantissement ressenti par un individu qui fait l'expérience d'une perte et a fortiori face à la mort ? Pour approcher ces questions, sinon y répondre, on peut prendre au mot la prétention principale des discours et pratiques consolatoires, celle d'apaiser. La question est celle du remède, de son efficacité comme de ses limites.

\section{La consolation philosophique et littéraire}

Le terreau naturel qui voit naître le discours de consolation est philosophique, et pour une raison indiscutable : la philosophie est la médecine de l'âme, qui dispense donc un certain nombre de remèdes ${ }^{8}$. Elle apprend comment se préparer à mourir, c'est-à-dire à bien vivre, à savoir faire face aux épreuves en les accueillant avec le plus de sagesse possible, grâce aux exercices spirituels qu'elle enseigne. L'essentiel pour le sage est de connaître des méthodes fiables sur lesquelles s'appuyer et de savoir les appliquer en cas de nécessité pour retrouver la tranquillité de l'âme, comme le préconisent les stoïciens mais aussi Épicure9.

7 En tant que genre et qu'interrogation existentielle, la consolation nous ramène donc aux sources même de la philosophie, aux questions les plus essentielles, celles qui conduisent à utiliser la raison et le savoir, mais aussi l'exercice de cette rationalité pour les mettre au service de la création d'une vie qui soit la plus libre possible. La 
consolation découle à cet égard de toutes les réflexions contenues dans les arts de bien vivre et de bien mourir, dans toute la littérature éthique qui va des stoïciens aux sceptiques. Elle s'inscrit dans la réflexion que suscite le deuil, quelle qu'en soit la gravité, et en tant que discours philosophique, le discours de consolation se focalise également sur la question pratique des actes. Dans une moindre mesure, elle fait écho par exemple aux débats sur l'immortalité de l'âme chez Platon. Il se pourrait que, d'après les sources fragmentaires dont nous disposons pour l'époque présocratique, se développe déjà une réflexion sur la sagesse qui aborde, de façon implicite, la consolation ${ }^{10}$. Comme l'a bien montré Pierre Hadot, les philosophies antiques «ont donc développé toutes sortes de pratiques de thérapie de l'âme, qu'il s'agisse de l'exhortation, de la réprimande, de la consolation, ou de l'instruction ${ }^{11}$ ".

8 Le premier texte consolatoire connu est une lettre, perdue, d'un philosophe académicien, Crantor de Soles ${ }^{12}$. Il n'est pas anodin que ce soit précisément la forme épistolaire qui serve de berceau au discours de consolation et demeure ensuite le support le plus employé. Le cadre épistolaire est tout particulièrement propice à l'expression d'une forme d'intimité, alors même que les marques du lyrisme y sont largement tributaires de normes rhétoriques et sociales bien balisées ${ }^{13}$. La lettre constitue la forme privilégiée où cohabitent les deux dimensions qui fondent la consolation : l'empathie face aux infortunes et les exhortations à se reprendre : deux élans relevant du devoir d'amitié. En outre, la lettre est un objet transactionnel puissant entre les épistoliers, elle peut, le cas échéant, consoler celui qui l'écrit comme son destinataire. Par sa matérialité même, qui pallie l'absence de l'autre, elle apporte $\mathrm{du}$ réconfort en prouvant, par sa seule existence, le lien allant du simple respect des normes sociales à l'attachement véritable, voire à l'affection.

9 Ces origines philosophiques nous éclairent sur les différentes formes que la consolation adopte au cours de l'histoire, dans les textes de circonstances comme dans la fiction. La consolation est un sujet philosophique qui s'invite tout naturellement dans la littérature, d'Homère aux auteurs actuels. Elle se situe à la lisière des thématiques autour de la souffrance, prises en charge notamment par la modalité élégiaque. Bien représentée dans l'épopée par exemple, l'expression de la douleur y est protéiforme. Chez Homère, la peine "subsiste au-delà de la consolation et du pardon ", comme le souligne Alain Michel ${ }^{14}$, en particulier dans le passage bien connu où Achille console Priam $^{15}$. Les poètes latins comme Virgile ou encore Ovide développent aussi de longs passages élégiaques qui abordent la consolation, qu'elle semble possible, évoquée directement ou remise en cause ${ }^{16}$.

Un autre aspect mérite d'être relevé. La consolation philosophique par excellence est celle qui vise à apporter un remède à la peur de la mort. Mais la mort n'est pas le seul objet de la consolation. L'exil, la perte des honneurs, la douleur, la maladie, la vieillesse, la ruine des cités constituent quelques-uns des thèmes de prédilection des traités moraux comme des lettres de circonstances et de la fiction. Comme le dit le titre de Pétrarque, il faut aussi trouver des «remèdes " à ces infortunes ${ }^{17}$. Cependant il n'est pas productif de classer les objets de déploration en fonction du seul critère trop peu efficient de leur universalité, la perte d'un proche étant considérée comme la forme la plus douloureuse et la plus topique. Il est par contre précieux de suivre l'évolution des raisons de consoler selon les époques. De ce point de vue, la consolation est un observatoire très éclairant pour appréhender les valeurs d'une société donnée et les pratiques mises en œuvre pour soulager la peine ou se préparer aux épreuves. Elle 
permet de saisir les spécificités historiques et éthiques d'une époque, ses aspirations et ses doutes. Il n'est pas anodin que l'Italie du $\mathrm{XV}^{\mathrm{e}}$ siècle se soit dotée de confréries de confortatori, ces associations laïques de miséricorde qu'a pu observer Montaigne ${ }^{18}$. Les traités d'épistolographies du XVII siècle présentent eux aussi, à côté de modèles de lettres attendues sur la perte des proches ou des honneurs, des sujets de consolations singuliers, en relation directe avec les besoins d'une société d'Ancien Régime soucieuse de se prémunir, par exemple, de la trop grande liberté prise par ses enfants ${ }^{19}$. Au travers des objets dont on console ses contemporains, se font sentir les principaux enjeux sociaux et philosophiques d'une civilisation, ses craintes les plus visibles et ses désirs de contrôle les plus palpables. La consolation agit là où le bât blesse.

11 Ainsi les objets de la consolation, les formes discursives reprises ou forgées pour les soutenir et les rituels sociaux fluctuent-ils de concert au contact de l'histoire pour en embrasser les réalités les plus saillantes ${ }^{20}$. Le protestantisme produit non seulement de nouveaux rituels d'accompagnement et de consolation aux malades mais aussi des formes discursives inédites ${ }^{21}$. Port-Royal illustre un usage de l'épître de consolation non moins remarquable. Ici, la lettre traduit toute l'ambiguïté du statut de la parole dans ce milieu conventuel singulier ${ }^{22}$.

Le discours consolatoire repose donc toujours sur une philosophie, quelqu'imprécise ou implicite qu'elle puisse sembler au lecteur moderne, et, en fait de remède, ce discours paraît à première lecture très confiant dans ses capacités à guérir, à apaiser la douleur. Il faut pourtant dépasser cette impression. Les grands textes consolatoires sont de l'ordre du souhait et même du virtuel, ils ont l'espoir, et non la certitude, que la parole puisse apaiser la douleur. Ils s'appliquent à relayer une forme de discipline, voire d'autodiscipline, puisqu'ils conseillent vivement à l'affligé de se détourner du chagrin ${ }^{23}$. En d'autres termes, ils relèvent pleinement d'une rhétorique, cette autre manière d'apporter un remède par les mots.

\section{La consolation rhétorique, entre deux types de discours}

13 Si la philosophie est la médecine de l'âme, la rhétorique intervient comme le médecin, par son art d'appliquer des mots aux maux ${ }^{24}$. Elle s'emploie à offrir des outils pour répondre à la variété plus ou moins infinie des situations humaines, car à chaque événement correspond un type de discours singulier et approprié, littéralement ajusté ${ }^{25}$. Les traités de l'époque moderne présentent ainsi une description de chaque type de discours suivie d'une trame à suivre (un plan en deux ou trois parties) ; Érasme ajoute à ces développements un florilège d'exemples développés ${ }^{26}$. Il va de soi que la consolation est très bien identifiée par les traités comme type de discours, à côté par exemple de l'oraison funèbre pour rendre hommage aux morts ${ }^{27}$. Or, le type de discours nommé consolation se rapproche d'un autre, la lamentation. Consolation et lamentation présentent un double dénominateur commun thématique et formel ${ }^{28}$ : leur comparaison est plus qu'instructive, elle est essentielle.

Sur le plan thématique, ces deux types de discours ont pour principal objet la douleur, mais là où la lamentation produit une forme de stase lyrique, un contrepoint pathétique qui emporte tout sur son passage ${ }^{29}$, suspend le temps tel, en musique, le grand solo du chanteur lyrique, la consolation se rapporte plus à la danse et plus 
précisément au pas de deux. En régime épistolaire tout particulièrement, le consolateur dialogue avec son interlocuteur affligé, se rapproche de lui tout d'abord pour mieux, dans un second mouvement, lui faire la leçon et l'entraîner sur son propre terrain, celui de la raison et du devoir ${ }^{30}$. La lamentation par contre est dominée par une seule voix qui se détache de son contexte et fait oublier toute idée de temporalité pour créer un moment de pur lyrisme, c'est pourquoi les monologues de plainte forment des extraits de texte facilement repérables.

De plus, les passions soulevées par ces deux genres reproduisent la différence de degré entre les émotions pathétiques et les émotions plus douces et plus durables, que Quintilien appelle aussi éthiques ${ }^{31}$. Cette différence d'intensité est à nuancer ou plus exactement à replacer sur le plan formel. La consolation comme la lamentation peuvent être des genres autonomes mais aussi des parties à l'intérieur d'autres discours, où elles forment une séquence spécifique. Le discours consolatoire intègre une section consacrée à l'expression de la plainte, au moment où le consolateur mesure la peine subie en évoquant les objets de la souffrance pour les magnifier au moyen d'un éloge. Lamentation et consolation sont aussi des modalités discursives, des genres traversés par d'autres types de discours: on en trouve des traces dans les oraisons funèbres et dans l'expression des condoléances ${ }^{32}$. (Se) lamenter et (se) consoler s'enchevêtrent. Mais la lamentatio induit une expérience émotionnelle qui, sublimant le malheur, nous aspire dans une verticalité absolue, alors que la consolation remet tout au contraire les pieds sur terre. Soit, pour reprendre le classement très éclairant des traités : la lamentation relève de l'épidictique, et la consolation, du délibératif et donc de l'action.

La consolation appartient pleinement à notre monde car dans l'acte de consolation, il s'agit de ramener à la vie d'ici-bas pour préparer la vie éternelle, à la rationalité et aux valeurs communes alors que la lamentation nous fait vivre, en temps réel et sous nos yeux ce moment de crise aux confins de l'humanité. La lamentation a une dimension spectaculaire, elle appartient à la poétique, la consolation est une affaire de regards où l'on attend de l'autre qu'il change de point de vue sur le malheur qui l'accable. Porteuse d'une intention et d'un mobile, elle relève de la rhétorique. Elle sait où elle va et conduit son interlocuteur sur le chemin de la vertu, là où la lamentation, quant à elle, semble tourner en rond et sur elle-même ${ }^{33}$. À l'inverse de la lamentation, la consolation demande de l'art, alors que "la douleur rend éloquent tout un chacun ", comme l'écrit Érasme et plus tard Vossius ${ }^{34}$.

L'art de consoler s'appuie sur la maîtrise d'une technique de persuasion qui en appelle à l'insinuation et à l'aptum. Il faut intervenir au bon moment, ce qui est un défi en soi. Vossius distingue deux méthodes pour consoler les âmes faibles, les fortes se dispensent de toute consolation. Le kairos est le correspondant philosophique de l' aptum ou decorum. La consolation ne saurait être frontale. Et elle doit s'ajuster à l'état d'esprit de l'affligé, répondre à la gravité de son affection. Depuis Ménandre, en passant par Érasme, Fabri, Scaliger ou encore Vossius, tous les traités préconisent un plan en deux parties pour consoler les âmes affligées. Les trois textes reproduits dans la partie «ATELIER » de ce numéro conseillent de commencer par s'assurer les bonnes grâces de l'affligé, surtout si celui-ci n'est pas doté d'une âme forte, avant de le consoler à proprement parler, par une exhortation à se défaire de son état mélancolique. Chaque civilisation se fait d'ailleurs une idée plus ou moins précise du moment où l'on doit 
cesser de pleurer ; ses injonctions s'appuient sur la nécessité de modérer les larmes et la phase de lamentation, afin d'éviter tout débordement ${ }^{35}$.

Revenons brièvement sur cette composition en deux parties. La première requiert de la douceur, mais une douceur sans mollesse, de celle qui tire vers la fermeté et le respect des règles de bienséance et de courtoisie, en un mot du lien social. Elle traduit une empathie concertée, prise dans un processus dont elle n'est qu'une étape. D'abord il y a l'approche, où l'on capte sinon la bienveillance du moins l'attention de l'autre, tel un médecin face au malade, car celui qui a besoin d'être consolé a un "jugement défaillant » comme l'indique Érasme. La deuxième partie se compose d'un discours parénétique émaillé de maximes philosophiques (ou de citations poétiques) ayant fait leur preuve et reflétant les croyances et les valeurs sociales. Ces deux phases pourraient se résumer cavalièrement par deux verbes: accrocher et reprocher, le second ne pouvant exister sans le premier. Cependant mesurer l'exacte ampleur du deuil vécu est en soi tout un programme qui dépasse la technique et requiert, comme la médecine, le consentement du malade. L'insinuation évoquée surtout par les traités du XVII siècle expose sans détour l'enjeu de cette captation : il faut créer un espace où se rencontrent les cœurs ${ }^{36}$. S'insinuer évoque une qualité de la parole, c'est celle qui s'adresse au cœur de l'autre, ce qui suppose d'avoir soi-même du cœur ou d'adopter une posture qui en apportera, par imitation, à celui qui n'en pas encore assez ou ne pas comment en avoir. C'est en somme faire preuve d'une forme de courtoisie chrétienne. Avec l'exercice consolatoire, les rhétoriciens livrent les secrets de leur art, et la voie à suivre grâce aux exemples qu'ils consignent. À destination de jeunes élèves appelés à savoir diriger et se diriger dans un proche avenir, les traités de rhétorique se font l'écho de nécessités sociales et politiques. Ils fournissent des modèles de discours et de comportements à suivre; leurs enseignements résultent d'une fine connaissance du fonctionnement de la psyché, ce que les métaphores médicales chez Érasme tout comme le lexique des émotions très présent dans leurs analyses montrent sans ambiguïté.

Liée à la lamentation, la consolation est ainsi un genre carrefour, elle-même traversée par d'autres types de discours, surtout les genres considérés comme funèbres. Construite sur le désir de toucher l'autre, d'atteindre son cœur pour le réconforter et surtout le ranimer et le ramener avec vigueur à la raison, elle est tantôt une pièce autonome, tantôt une partie exportable dans des environnements qui lui sont proches, comme la plainte ou l'éloge, parties que l'on retrouve aussi dans les oraisons funèbres et bien entendu dans les lettres par exemple. Pièce autonome ou partie d'un tout, la consolation exige un examen méticuleux en contexte et une appréhension de grande ampleur puisqu'elle est pensée en fonction d'une série d'autres actions rhétoriques avec lesquelles elle interfère en continu. Si elle est, philosophiquement parlant, un remède pour apaiser la douleur, en pratique c'est un remède qui nécessite tout l'art et le tact du médecin.

\section{Des maux aux mots : Les limites de tout discours de consolation}

20 Le remède n'est pas toujours efficace. Depuis l'épopée, les lecteurs savent que la souffrance des héros peut être inaccessible au raisonnement. Aucun discours de consolation ne peut les atteindre. Face au discours philosophique qui voit dans la 
parole un médicament doté du pouvoir de guérison, les grandes figures du patrimoine culturel européen représentent un " degré de deuil » qui épuise "les derniers efforts » de l'art et incarne une douleur d'une profondeur abyssale, comme l'écrit Montaigne ${ }^{37}$. Ce dernier réfute d'ailleurs l'idée même de la consolation, pour lui préférer la diversion ${ }^{38}$. Si les figures de héros inconsolables abondent dans la littérature européenne, tous ne repoussent pas l'idée ou l'espoir d'être consolé.

Hamlet, mais aussi dans une moindre mesure et animé par d'autres mobiles le je du Canzoniere de Pétrarque, incarnent un refus de la consolation ou d'être consolé ${ }^{39}$. Le premier rejette le discours consolateur car mensonger de son tout nouveau beau-père, quant au second, il demeure longtemps sourd à l'appel d'en haut qui l'invite à transformer son amour pour Laure en amour pour Dieu Ces deux exemples suffisent à donner une idée assez précise du conflit intérieur qui peut surgir lorsqu'un personnage est en proie à une douleur qui le surpasse et qu'on le montre ou qu'il se montre luimême incapable de sortir des griffes du deuil ${ }^{40}$.

Les résultats que nous proposons dans ce dossier d'articles rendent tangibles les limites de tout discours consolatoire. La pratique, les rites comme l'exercice de la consolation rencontrent des résistances dont certaines correspondances privées portent les traces. Si les manuels d'épistolographie du Grand Siècle en particulier pourraient laisser à penser que le discours consolatoire est toujours agencé selon un strict respect des usages protocolaires, faisant usage d'un déploiement de moyens rhétoriques ostentatoires, dans la pratique, il en va tout autrement ${ }^{41}$. De nombreuses correspondances présentent des lettres de consolation assez brèves ${ }^{42}$. En outre, lorsqu'on écrit à un proche, on peut répondre à son désir de consolation par l'expression d'une plainte infinie. Sans que la consolation soit rejetée en bloc, il arrive que certains affligés, alors même qu'ils tiennent compte des normes épistolaires qu'ils partagent avec leur correspondant, expriment les difficultés qui les tenaillent malgré tout et qui rendent toute consolation inaudible. Ce motif se retrouve par exemple dans la correspondance d'un jeune veuf inconsolable qu'analyse Paula Barrros ${ }^{43}$. Ses lettres de réponse aux consolations reçues font partager à ses destinataires le manque de tact de ses amis vis-à-vis de son chagrin, ce dont, paradoxalement, il s'excuse et se plaint tout à la fois. Le thème du deuil inconsolable se retrouve également chez un grand parlementaire français. Dans la dernière édition de son œuvre complète, Guillaume du Vair publie une longue missive adressée à sa sœur défunte. Il confie que les techniques et les raisonnements ordinaires ne parviennent pas à le consoler de cette disparition ${ }^{44}$. $\mathrm{Au}$ récit des derniers instants de sa sœur succède une longue plainte que seul l'espoir de lui rendre hommage par l'écriture de cette épître semble pouvoir atténuer. La résistance à l'égard des lieux communs, en général exposés dans la partie exhortative des discours consolatoires, transparait sous forme de dialogue dans le récit de la dernière nuit avant l'exécution d'un de ses proches amis que rédige Della Robbia, un noble humaniste florentin au tout début du XVI ${ }^{\mathrm{e}}$ siècle ${ }^{45}$.

Sans faire directement écho au texte de Lucien intitulé Sur le deuil, où la mort se moque vertement des pratiques mortuaires en ridiculisant l'attitude des humains, ces quelques exemples significatifs qui traduisent les difficultés éprouvées à se conformer aux règles de bienséances sociales et rhétoriques nous montrent sans ostentation le poids de pratiques prises à la lettre et quelquefois mal interprétées par des consolateurs zélés mais maladroits. Ils dénoncent les efforts stériles d'une parole à trouver la voie des cœurs, et traduisent l'amertume ou la colère, voire la culpabilité d'endeuillés qui 
revendiquent le droit de pleurer plus que la coutume ne les y autorise, et le droit à être entendus. Dans ces textes, il se trouve que c'est moins la parole du consolateur, normée, construite, répondant à une intention unique - ramener l'apaisement d'autrui et conserver la paix sociale - qui est remise en question que l'absence de synchronicité suffisante entre le deuil éprouvé et la parole de réconfort. La question du kairos apparaît centrale. Les traités de rhétorique d'Ancien Régime construisent leur commentaire sur le discours de consolation autour de cette question du temps, car le tact, cet effet du decorum nécessaire à tout bon orateur, est toujours une affaire de temporalité, de tempo et de juste mesure ${ }^{46}$. Le tact est, de nouveau, une question de médecin : la consolation comme remède demande tout un art, surtout face à un malade qui se proclame incurable - en l'occurrence : inconsolable.

Ces quelques mots de présentation auront montré la nature vivante d'un corpus sur la consolation, sujet qui excède la littérature alors même qu'il en est profondément imprégné. Ces premiers résultats esquissent les grandes lignes du territoire occupé par la consolation et disposent quelques balises : la nature philosophique de tout discours consolatoire, sa place dans les belles lettres et dans les écrits non fictionnels, le regard critique porté sur un objet qui fait débat. Présente à l'intérieur du champ littéraire comme à ses confins, pouvant devenir à l'occasion un théâtre des plaintes qui découvre le jeu des infortunes, la consolation excède les frontières du poétique, de la rhétorique, du textuel, du littéraire et du rituel. Elle les dépasse mais d'une certaine façon les réconcilie, en montrant encore une fois la pertinence d'ouvrir notre champ de vision afin de couvrir le plus vaste espace possible, en diachronie comme en synchronie, pour faire tenir ensemble un corpus multiforme qui se déploie dans et hors du texte tout en nous y ramenant toujours. Plus encore que par l'iconographie, la pratique consolatoire est portée par les témoignages textuels variés qui en donnent une représentation tout à la fois normée, contrastée et largement discutée. Discours codifié, la consolation suppose l'efficacité thérapeutique de la parole en association avec les gestes. Elle est enseignée dans les traités de rhétorique comme dans les traités d'épistolographie. Savoir consoler un proche ou un grand de ce monde ouvre des portes et maintient le lien social. Plus ou moins fixés dans des descriptions rhétoriques qui se font écho les unes les autres, progressivement tenus à distance par les manuels d'épistolographie du Grand Siècle, les discours sur la consolation sont des indices précieux de la façon dont une société appréhende les liens entre les individus, dont elle éduque à bien vivre, protège, se défend et corrige. La consolation a bien une répercussion politique car le consolateur n'est pas seulement un médecin, mais aussi un conseiller prudent.

Ce sujet nous invite ainsi à reconsidérer l'interaction entre les règles et les pratiques, à nous interroger pour savoir dans quelle mesure les conseils de composition édictés par les manuels de rhétorique et d'épistolographie ou par les manuels d'accompagnement aux affligés sont de simples supports à partir desquels on adapte et on invente, ou au contraire on se détache de facto des usages et des modèles qui ne cessent de se modifier en surface ou en profondeur. Nous découvrirons peut-être que dans ces sources, les règles enseignent en réalité plus qu'il n'y paraît l'esprit de la consolation, sa philosophie.

Fenêtre ouverte sur des pratiques du soin apporté à l'autre, les résultats proposés dans ce numéro apportent des surprises. La consolation, au moins jusqu'à la fin du XVII siècle, nous guide pour penser ensemble deux actions que l'on aurait tendance à séparer, prendre soin et exhorter, et à dissocier deux formes que l'on a tendance à 
amalgamer, la plainte et la consolation ${ }^{47}$. Formulés ainsi pourtant, empathie et encouragement rappellent à la mémoire un vieux proverbe : qui aime bien châtie bien. C'est que dans les sociétés où règne la rhétorique, s'attendrir avec l'autre sur son chagrin, manifester son empathie, louer l'objet perdu, c'est aussi et déjà vouloir qu'il retrouve à nouveau le bon chemin, celui où son cœur se remet à battre à son propre rythme puis au rythme des autres et de la collectivité humaine où il accepte de se faire rattraper par la vie.

\section{NOTES}

1. Titre du fameux "témoignage " de Stig Dagerman, Notre besoin de consolation est insatiable, traduit du suédois par Ph. Bouquet, Arles, Actes Sud, 1981 (traduction de Värt behov av tröst, Stockholm, Norstedts, 1955). Voir plus récemment le roman de Gr. Ehrlich, La consolation des grands espaces (Paris, Albin Michel, 1996, traduction de The Solace of Open Space, Viking Penguin, $1985)$; A. Gavalda, La consolante (Paris, J'ai Lu, 2008) ; le témoignage de la journaliste F. Flament, La consolation, Paris, Lattès, 2016, ou encore Y. Jablonka, qui, dans un travail d'historien de la mémoire, rédige une «biographie familiale » en quête d'une forme de consolation (Histoire des grands-parents que je n'ai pas eus, Paris, Seuil, 2012).

2. Pour une analyse comparant les théories de Freud et l'ancienne rhétorique, voir Th. Carr, «L' èthos dans les rhétoriques de consolation de l'Ancien Régime à la lumière des perspectives modernes sur le deuil ", in F. Cornilliat et R. Cooper dir., Èthos et pathos. Le statut du sujet rhétorique, actes du Colloque international de Saint-Denis (19-21 juin 1997), Paris, H. Champion, 2000, p. 339-348. Voir aussi le bémol apporté par M. Fœssel, Le temps de la consolation, Paris, Seuil, 2015.

3. Sénèque, Consolations, I. Hadot éd., Paris, Rivages poche/Petite Bibliothèque, 1992.

4. Dans le langage courant et la vie quotidienne, comme dans la fiction contemporaine. Le roman de D. Foenkinos, La délicatesse (Paris, Gallimard, 2009) ou encore celui d'E. Carrère, D'autres vies que la mienne (Paris, POL, 2009) ne présentent pas de véritable discours consolatoire face au deuil des personnages principaux. La consolation s'exprime plutôt par une forme d'empathie et par un élan spontané pour soutenir autrui. Les méthodes consolatoires à disposition aujourd'hui, dites modernes, émanent plutôt de techniques de communication ou relèvent de la psychologie, dans une moindre mesure peut-être des religions. Voir J. Attali et S. Bonvicini dir., Consolations, Paris, Librairie Arthème-Fayard/Pluriel, 2017, ouvrage issu de la série d'émissions «Le sens des choses » sur le thème de la consolation, diffusée sur France Culture en août 2010.

5. Les articles de ce numéro (à l'exception de celui de C. Lignereux) constituent les premiers résultats d'une enquête menée par un groupe de chercheurs lors de plusieurs journées d'études sur la consolation de l'Antiquité grecque à la fin du XVII ${ }^{\mathrm{e}}$ siècle, organisées et animées par C. Martin-Ulrich. Les différents programmes de ces journées sont disponibles à l'adresse suivante : http://www.ircl.cnrs.fr.

6. Une part conséquente de ces sources demeure d'ailleurs cachée à nos propres yeux puisque qu'elles ne sont pas lues à la lumière de cette question et l'on sait bien que c'est le regard qui crée l'objet. Voir à ce sujet, infra, la lecture du poème de Lucrèce par S. Luciani, «Lucrèce et la tradition de la consolation ".

7. Voir la partie ATELIER où Vossius envisage la position du consolateur en fonction de son lien avec l'affligé, soit inférieur, soit supérieur, soit égal. 
8. Voir A.J. Voelke, La philosophie comme thérapie de l'âme. Études de philosophie hellénistique, Fribourg, 1993 ; J. Pigeaud, La maladie de l'âme. Étude sur la relation de l'âme et du corps dans la tradition médico-philosophique antique, Paris, Les Belles Lettres, 1981, ou encore D. Lucas, « La philosophie antique comme soin de l'âme », Le Portique [En ligne], 4-2007 | Soin et éducation (II), mis en ligne le 14 juin 2007, consulté le 30 septembre 2016. URL : http://leportique.revues.org/ 948

9. Voir Les Stoïciens, éd. É. Brehier, Paris, Gallimard (Pléiade), 1962 ; P. M. Morel, Épicure, la nature et la raison, Paris, Vrin, 2009.

10. Voir Penseurs grecs avant Socrate, de Thalès de Milet à Prodicos, éd. J. Voiquin, Paris, GF, 1964.

11. P. Hadot, Qu'est-ce que la philosophie antique ?, Paris, Folio essais, 1995, p. 330, citant ibid., note 1 J. de Romilly, " Patience mon cour ! », L'Essor de la psychologie dans la littérature grecque antique, Paris, Les Belles Lettres, 1984.

12. Voir dans ce numéro, la note 36 de l'article de S. Luciani, "Lucrèce et la tradition de la consolation ». Ce philosophe du début du $\mathrm{III}^{\mathrm{e}}$ siècle a rédigé une lettre de consolation à un ami pour apaiser son chagrin au moment de la perte de son fils. Ce texte a servi de modèle et de référence, à commencer par Cicéron.

13. Pour l'époque moderne, mais applicable aux textes antiques, voir C. Lignereux, " L'art épistolaire de l'âge classique comme champ d'application du savoir rhétorique ", Exercices de rhétorique [En ligne], 6 | 2016, mis en ligne le 11 février 2016, consulté le 12 février 2016. URL : http://rhetorique.revues.org/441; DOI : 10.4000/rhetorique.441. Sur les liens entre genre épistolaire et consolation, voir P. Fleury, Lectures de Fronton, un rhéteur latin à l'époque de la Seconde Sophistique, Paris, Les Belles Lettres, 2006, p. 65-77 ; D. J. Ochs, Consolatory Rhetoric: Grief, Symbol and Ritual in the Greco-Roman Era, Columbia, University of South Carolina Press, 1993.

14. A. Michel, « Sagesse et humanité », dans E. Huisman-Perrin dir., La consolation. Mots pour maux, Paris, éditions Autrement, 1997, p. 124-137 (p. 124).

15. Iliade, chant 24, v. 518-551.

16. Notons une avancée remarquable des études sur la consolation portant sur l'Antiquité par rapport aux autres époques : parmi les ouvrages récents, voir H. Baltussen dir., Greek and Roman Consolations. Eight Studies of a Tradition and its Afterlife, Swansea, The Classical Press of Wales, 2013. Pour le premier Moyen Âge, et comme le rappelle M. Gally (voir infra, « Parler à l'autre, parler de soi : la consolation selon Abélard et Héloïse »), le corpus consolatoire ne fait pas directement écho à la tradition antique de la consolation. Cependant les principales formes poétiques telles que les complaintes, la malmariée, peut-être l'aube où l'amant déplore l'arrivée du jour, les rondeaux ou les ballades, en un mot l'ensemble de la lyrique médiévale mériteraient d'être revisitées. Pour une définition du genre de la plainte, voir $\mathrm{Cl}$. Thiry, La plainte funèbre, Turnhout, Brepols, 1978.

Le texte de Boèce innerve toute la pensée médiévale et philosophique de la période médiévale et bien au-delà. Thomas More s'en est inspiré pour rédiger son Dyalogue of Conforte againste Trybulacion comme le souligne J.-Y. Tillette dans son introduction à la Consolation de Philosophie, éd. Cl. Moreschini et É. Vanpeteghem, Paris, Le Livre de poche, 2005, p. 10.

Voir J. Vignes, « la lettre de consolation de Plutarque à sa femme traduite par La Boétie et ses prolongements chez Montaigne, Céline et Michaël Fœssel », infra, dans la partie ANALYSE de ce numéro.

17. Voir par exemple la belle liste d'infortunes dressée par Pétrarque dans Les remèdes aux deux fortunes, livre II, volume 1, trad. Ch. Carraud, Grenoble, Jérôme Million, 2002 et les listes des traités de rhétorique dans la partie ATELIER de ce numéro. Les traités d'épistolographie présentent aussi des listes remarquables. Voir C. Lignereux, op. cit.

18. Voir infra, l'analyse et les conclusions de l'article d'E. Taddia, «Les mots et les actes. Consoler le condamné à mort en Italie à la Renaissance : la Recitazione de Luca Della Robbia (1513)». 
19. Voir, dans les Essais morales de Nervèze (Les CEuvres morales du sieur de Nervèze, Antoine du Brueil, Paris, 1610 ; p. 394), l'exemple d'une lettre de consolation «à un gentilhomme sur la réduction de son fils dans un cloistre ».

20. Voir infra, l'analyse de P. Desmoulière sur les liens entre commémoration, éloge et consolation dans « La consolation dans les tombeaux poétiques en Italie, en France et dans les îles britanniques (1590-1640)».

21. Voir parmi les récents ouvrages, V. Ferrer, Exercices de l'âme fidèle. La littérature de piété en prose dans le milieu réformé francophone (1524-1685), Genève, Droz, 2014 analysant entre autres la création d'un sociolecte spécifique : la langue de Canaan et les corpus de sommes spirituelles émanant des manuels évangéliques qui s'épanouissent dès la seconde partie du XVI siècle; parmi les nombreux articles de M. Carbonnier-Burkard, voir " Un manuel de consolation au XVII siècle : les Visites charitables du pasteur Charles Drelincourt», Bulletin de la Société de l'Histoire du Protestantisme Français, t. 157, 2011, p. 331-356 ; voir aussi infra, Ch. Bernat, « Désolation, vocation, exemplarité. Les lettres de consolation de Théodore de Beringhen (1686-1700)». Pour les îles britanniques, voir P. Barros, "Appréhender les émotions au travers des rituels religieux? L'exemple des visites des malades chez les protestants anglais, c. 1559-1640», disponible à l'adresse : http://britaix17-18.univ-provence.fr/texte-seance3.php.

22. Voir infra, A. Cousson, "Je prends part à votre douleur". La lettre de consolation à PortRoyal ».

23. Nous songeons aux analyses de N.Loraux (Les mères en deuil, Paris, Seuil, 1990), qui concernent la société grecque ancienne, mais peuvent être appliquées à d'autres contextes.

24. C'est justement le sous-titre d'un recueil d'articles: E. Huisman-Perrin dir., La consolation. Mots pour maux, éditions Autrement, Paris, 1997.

25. Voir Fr. Goyet, «Le problème de la typologie des discours ", Exercices de rhétorique $1 \mid 2013$, URL: http://rhetorique.revues.org/122; et Ch. Noille, « Les genres du discours dans l'ancienne rhétorique : listes, schémas et mode d'emploi, avec un exemple (le discours de Germanicus) », Exercices de rhétorique, 3 | 2014, URL : http://rhetorique.revues.org/337.

26. Ch. Noille définit ce florilège (silvae) comme un « recueil de matériaux argumentatifs extraits d'œuvres modèles ». Voir la section ATELIER.

27. Sur la lettre de consolation, voir A. Tarrête op. cit. et N. Carabin, notamment, « Les lettres de consolation de Nicolas Pasquier: la lettre de consolation ", Revue d'histoire littéraire de la France, 2002/1, vol. 102, p. 15-31. Sur les lettres de condoléances, voir Th. Carr, «Se condouloir ou se consoler? Les condoléances dans les manuels épistolaires de l'Ancien Régime », French Language and Literature Papers, 1997, p. 217-235 et C. Lignereux, "Une routine de la civilité épistolaire: l'expression de la condoléance », Exercices de rhétorique [En ligne], 6 | 2016, mis en ligne le 08 février 2016, consulté le 02 octobre 2016. URL : http://rhetorique.revues.org/437.

28. Sur la plainte, voir le numéro intitulé «La plainte aux XVI ${ }^{\mathrm{e}}-\mathrm{XVII}{ }^{\mathrm{e}}$ siècles, ou la littérature comme lien » de la revue en ligne L'esprit Créateur (parution prévue en juin) dont nous n'avons pu lire à ce jour que la présentation d'Hélène Merlin-Kajman. Dans ce texte, l'auteure évoque à fort juste titre l'incompatibilité qui existe entre plainte et consolation et les frictions qui se créent entre ces deux genres : https://espritcreateur.org/forthcoming-issues. Voir aussi F. Alazard dir., La plainte à la Renaissance, journées d'études des 16 et 17 novembre 2005, Paris, H. Champion, 2008. Voir aussi le distinguo entre « déploration » et « confort » dans la première partie de la thèse d'Ellen Delvallée, Poétiques de la filiation: Clément Marot et ses maîtres, Jean Marot, Jean Lemaire et Guillaume Cretin, dir. Fr. Cornilliat et Fr. Goyet, Rutgers University et Université Grenoble Alpes, 23 juin 2017.

29. C'est le sens grec du mot monodie.

30. Voir infra, C. Lignereux, op. cit., qui analyse une série de lettres de Mme de Sévigné portant sur la mort d'un des très jeunes enfants de Mme de Grignan où l'épistolière distille habilement les arguments de la consolation. 
31. Quintilien, Institution oratoire, VI, 2, 8-10, où évoquant les deux sortes d'émotions qui existent, il traduit le grec pathos par le latin adfectus, et ethos par un équivalent qu'il juge impropre mores. Pour bien les départager, il préfère les décrire en disant que «les premières commandent » et sont de courte durée, les autres « persuadent » et sont continus.

32. Voir A. Régent-Susini, "La "douleur publique" des oraisons funèbres, une douleur sans plainte? ", à paraître dans le numéro en ligne de la revue L'esprit Créateur, op. cit.

33. D'où l'usage du rondeau pour traduire la déploration (de Charles d'Orléans à Marot). C'est la raison pour laquelle Vossius considère que la lamentation nécessite moins d'art (Rhetorices contractae..., II, 19, § 14). C'est qu'elle ne relève pas de l'art rhétorique au même titre que les autres genres de discours, même si elle peut devenir un instrument de persuasion et même si elle utilise à plein les ressources de l'art, c'est-à-dire les arguments classés parmi les procédés d'amplification pour mettre en scène, dans la partie narrative, l'accumulation des malheurs et leurs effets. Sur l'amplification, voir S. Macé, "L'amplification, ou l'âme de la rhétorique. Présentation générale », Exercices de rhétorique [En ligne], 4 | 2014, mis en ligne le 05 décembre 2014, consulté le 06 décembre 2014. URL : http://rhetorique.revues.org/364

34. Érasme, De conscribendis epistolis (1522), éd.J.-Cl. Margolin, Opera Omnia Desiderii Erasmi Roterodami, Ordinis Primis, Tomus Secundus, Amsterdam, North-Holland Publishing Company, 1971, p. 555 (LB I 472) : «vnumquemlibet dolor soleat reddere disertum»; repris par Vossius, Rhetorices contractae..., II, 19, § 14 . Voir Cl. Martin-Ulrich, «La lamentatio : un discours sans art? Une enquête d'Érasme à Gerard Vossius ", in Les représentations de la souffrance. Actes du Colloque international des 12 au 14 mai 2016, Université Paris-Est Créteil, B. Petey-Girard et P. Severac dir., Paris, Garnier, 2017 (à paraître).

35. Voir Th. Carr, «La perte de l'autre et l'auto-consolation », in L'autre au XVII ${ }^{e}$ siècle. Actes du $4^{\text {ème }}$ colloque du Centre international de Rencontres sur le CVII ${ }^{\mathrm{e}}$ siècle, University of Miami 23 au 25 avril 1988, Biblio 17-117, 1999, p. 367-373. Et A.P. Barros, Les vivants à l'épreuve du deuil. Discours théoriques et écrits de circonstance en Angleterre, c. 1550-1640, thèse soutenue en 2007, en cours de publication, Paris, Garnier.

36. Voir S. Macé, « L'amplificatio, l'attenuatio et l'exercice de la conciliation : l'exemple des lettres et poèmes de consolation du premier XVII ${ }^{e}$ siècle ", Cahiers du GAGDES n¹3, 2013, p. 125-138.

37. Montaigne, Essais, I, 2, « De la tristesse ", éd. P. Villey, Paris, Quadrige, Presses universitaires de France, 1998, (1 ère éd. 1965), p. 12, citant la fameuse figure de Niobé. Il souligne bien que certaines douleurs ne sont pas représentables.

38. Ibid., III, 4, p. 830-839.

39. Shakespeare, Hamlet, I, 2. Sur Pétrarque, voir G. W. McClure, Sorrow and Consolation in Italian Humanism, Princeton, Princeton University Press, 1990 et S. Stroppa, Petrarca e la morte, Tra Familiari e Canzoniere, Rome, Aracne, 2014.

40. Condamné à subir les assauts de plusieurs personnages maléfiques, l'« écolier de Mélancolie » de Charles d'Orléans connaît aussi les affres d'un désespoir d'où la consolation semble absente. Cette expression sert de titre au recueil de ballades et de rondeaux du prince poète, réunis par Cl. Galderisi, dans son édition, L'écolier de mélancolie, Paris, Le Livre de poche, 1995.

41. Voir infra les conclusions de l'article de C. Lignereux, «Des prototypes rhétoriques à leur fragmentation épistolaire : l'exemple d'une consolation en pièces détachées ».

42. Ces corpus seront étudiés ailleurs, prochainement.

43. P. Barros, " "Piety to a dead man": les limites de la consolation dans la pratique épistolaire de Sir Kenelm Digby (Angleterre, 1633)».

44. G. du Vair, Les ceuvres de messire G. du Vair, Paris, Sébastien Cramoisy, 1641, « Sur la mort de Ph. Du Vair sa sœur », p. 669-675. A. Tarrête, « La Consolation de Guillaume Du Vair sur la mort de sa sœur (1584) ", dans Jean Balsamo dir., Les Funérailles à la Renaissance, Genève, Droz, 2002, p. 499-516.

45. Voir E. Taddia, op. cit. 
46. Pour les sources antiques de la consolation voir S. Luciani, op. cit.; et l'ATELIER, note 1, mentionnant Ménandre le rhéteur. Ajoutons Ps Démétrios de Phalère, Types épistolaires (voir l'édition moderne de P.-L. Malosse, Lettres pour toutes circonstances, Paris, Les Belles Lettres, 2004). 47. Il n'est pas anodin que presque au même moment, deux numéros de revue en ligne donnent à lire des analyses sur la consolation d'une part et sur la plainte de l'autre ; voir note 28.

\section{AUTEUR}

\section{CLAUDIE MARTIN-ULRICH}

Université de Pau et des Pays de l'Adour / IRCL - UMR 5186 